\title{
Energy-Aware Routing Hole Detection Algorithm in the Hierarchical Wireless Sensor Network
}

\author{
Najm Us Sama ${ }^{1}$, Kartinah Bt Zen ${ }^{2}$, Atiq Ur Rahman ${ }^{3}$, Aziz Ud Din ${ }^{4}$ \\ Faculty of Computer Science and Information Technology, Universiti Malaysia Sarawak, 94300 Kota Samarahan, Sarawak ${ }^{1}$ \\ College of Computer and Information Sciences, Jouf University, Saudi Arabia ${ }^{1}$ \\ Faculty of Computer Science and Information Technology, Universiti Malaysia Sarawak, 94300 Kota Samarahan, Sarawak ${ }^{2}$ \\ Faculty of Computing and Information Technology, Northern Border University, Rafha, Saudi Arabia ${ }^{3}$ \\ Shaikh Zayed Islamic Center, University of Peshawar, Pakistan ${ }^{4}$
}

\begin{abstract}
To minimize the communication overhead with the help of optimal path selection in Wireless Sensor Network (WSN) routing protocols is the challenging issue. Hierarchical routing optimizes energy utilization by distributing the workload among different clusters. But many-to-one multi-hop hierarchical routing will result in the excessive expenditure of energy near the sink and leads to early energy exhaustion of the nodes. Due to this, the routing hole problem can be caused around the base station. Data routed along the hole boundary nodes will lead to premature exhaustion of energy. This will maximize the size of the hole in the network. Detection of holes saves the additional energy consumption around the hole and minimize the hole size. In this paper a novel energy efficient routing hole detection (EEHD) algorithm is presented, on the detection of routing hole, the periodic re-clustering is performed to avoid the long detour path. Extensive simulations are done in MATLAB, the results reveal that EEHD has better performance than other conventional routing hole detection techniques, such as BCP and BDCIS.
\end{abstract}

Keywords-Wireless sensor network; routing protocol; hierarchical routing; routing hole problem; routing hole detection

\section{INTRODUCTION}

Routing in sensor networks is an active research topic and the responsibility of routing protocol is to enable the sensor nodes to select routes among the source node and sink node [1]. Energy balancing is always the main goal in sensor networks. Some applications might be very harsh, and very unlikely to renew or recharge the batteries of nodes in the sensor network. Thus, in a sensor network is non-renewable battery provision is the most challenging issues. It is a dire need to develop energy-efficient routing strategies that minimize power requirements at the overall network. Because unbalanced energy consumption will lead to routing hole problems, which is one of the most challenging issues in the communication process. Routing hole acts as an obstacle to communication and also known as "communication voids" [2, 3] and results in network partitioned. Much research has been done for detection, avoidance, and mitigation of routing hole problems [4-7].

Many kinds of literature used the cluster based technique to achieve energy efficiency and energy balancing [8-10] but in multi-hop clustering, the cluster heads have to transmit its own data as well as forward the data of other CHs. Due to the dual load, the lifetime of $\mathrm{CH}$ becomes lesser than others and leads to routing hole problem. Data routed along the hole boundary nodes will lead to premature exhaustion of energy of hole boundary nodes. This will maximize the size of the hole in the network. Detection of holes saves the additional energy consumption around the hole and minimize the hole size. It assures longer network lifetime and efficient use of nodes. Routing hole detection is the only solution to avoid the long detour. The routing hole should be detected in advance before to start the data transportation phase, and the nodes placed on the boundary of the hole announced the hole information to its neighbor nodes. Hence the long detour path can be avoided.

A novel energy efficient routing hole detection (EEHD) algorithm is presented, which compare the energy of cluster head $(\mathrm{CH})$ with a threshold value and advertises itself as a critical node if the energy is equal or lower than the threshold. And the critical node is known as the routing hole in a network. When the routing hole is detected the algorithm performs periodic re-clustering to avoid the long detour path. The causes of routing hole are presents and some of the strategies from research for routing hole detection, avoidance and mitigation are also discussed.

The rest of the paper is categorized as follows. The background and causes of routing hole problems have been presented in Section 2. A novel routing hole detection algorithm is illustrated in Section 3. Section 4 presents the performance discussion. At last, the conclusion is summarized in Section 5, followed by References.

\section{Routing Hole Problem}

In literature, existing routing protocols may lead to routing hole problem due to some inefficiency of routing technique. A routing hole consists of an area in the sensor network where a group of sensor nodes stop functioning or disconnected from the network and do not participate in the routing of the data [11]. The routing holes are caused by the failure of sensor nodes such as defect, battery exhaustion or external event damage.

\section{A. Causes of Routing Hole Problem}

The routing holes can be caused by a few reasons, which are the failure of sensor nodes in a random deployment, lack of coverage and inefficient routing protocol. 


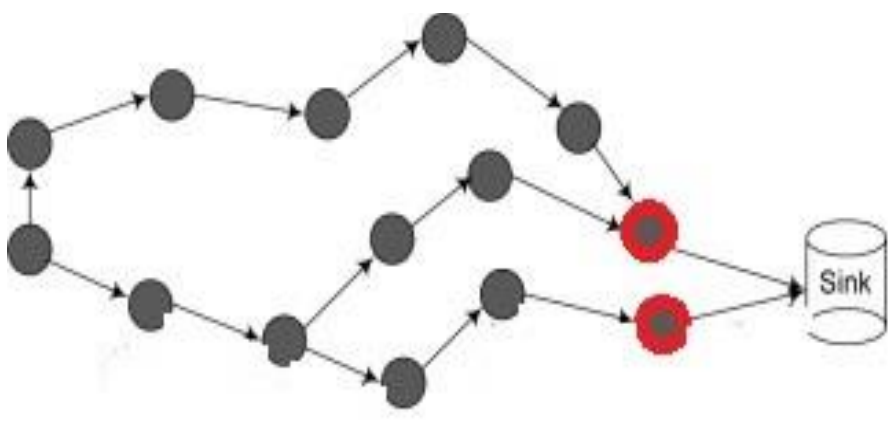

Fig. 1. Routing Hole Due to Random Deployment.

In random deployment, many-to-one multi-hop data routing pattern results in quicker loss of energy of nodes near the sink; this is referred to as routing hole problem.

As shown in Fig. 1 random deployment of nodes in wireless sensor networks, may lead to serious coverage overlapping among the nodes and due to critical nodes the network may suffer coverage hole problems. The presence of coverage-hole in the network area means that every point in the interested region is not being covered[12]. Coverage hole problem causes the network partitioned which leads to routing hole problem. In Fig. 2 the sensor node 2 is dying out, the area is now not covered by any sensor nodes and sensor node 1 cannot send its sense data to sensor node 3 .

Routing holes also can be caused by energy unaware routing as shown in Fig. 3. In multi-hop cluster based routing algorithm the sensor nodes send its sense data to $\mathrm{CH}$, then $\mathrm{CH}$ will send the aggregated data to its neighboring $\mathrm{CH}$ and so on till the packets received by the sink. But routing hole may occur because the $\mathrm{CH}$ near to base station has a maximum workload or due to worst path selection routing technique [13].

As regards the above-mentioned important challenge in WSNs, avoiding the routing hole problem is considered to be a crucial challenge for such networks and should definitely be taken into account. Many types of research have done on routing hole mitigation [14-23].

Routing hole detection is important because data routed along the hole boundary will lead to early exhaustion of energy at the boundary nodes. Early detection of holes avoids the additional energy consumption around the hole boundary and minimize the hole size. This pro-active early detection assures long network lifetime and nodes to be more energy efficient.

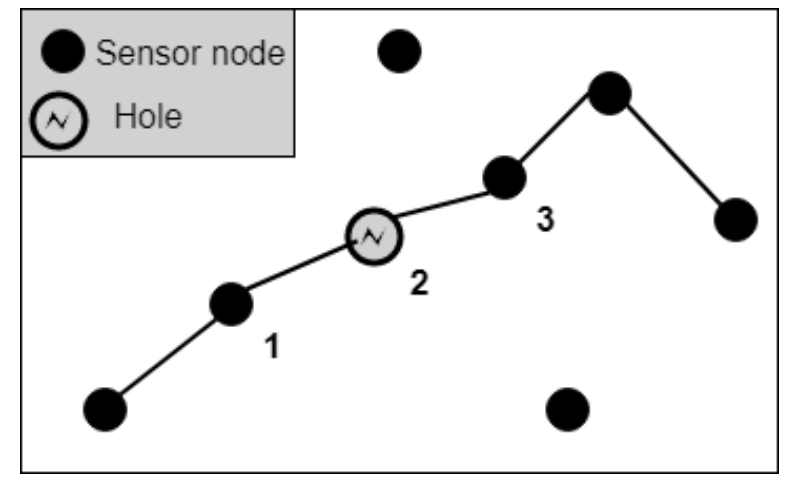

Fig. 2. Routing Hole Due to Lack of Coverage.

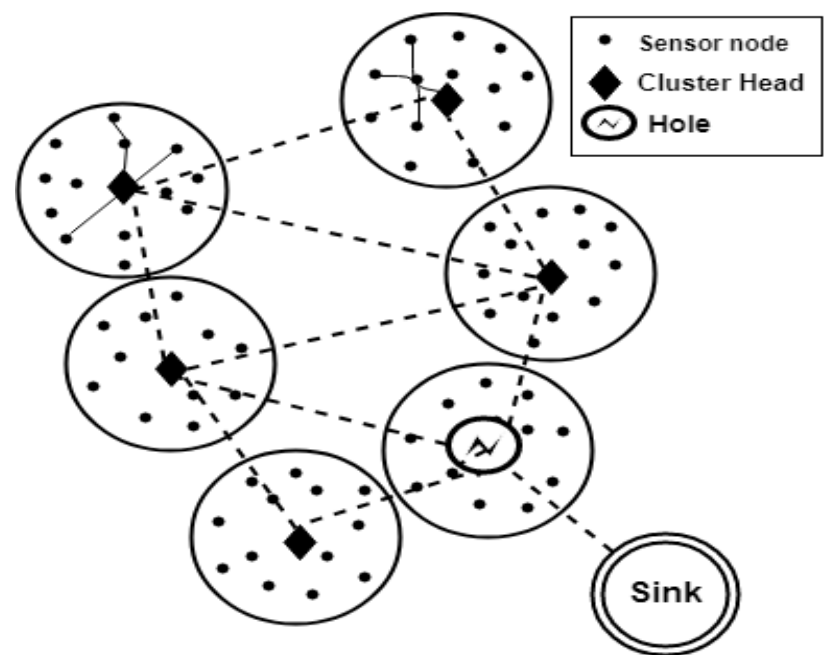

Fig. 3. Routing Hole in Multi-Hop Hierarchical WSN.

Authors in [24] proposed a distributed solution to detect the holes and their boundaries (BDCIS) in WSN. In the first step, each node collects connectivity information and builds its one-hop neighbors graph. In the second step independent sets are established, and then connect the independent sets in order to choose the best path in the last step. The simulation shows that this algorithm achieves enhanced performance in terms of minimum energy depletion, accuracy, and less communication overhead. Each node needs to share the connectivity information to construct independent sets from one-hop neighbor's graph, which make the proposed solution inefficient in terms of energy consumption. Coverage Protocol (BCP) is presented by authors in [25] that preserves both border and area coverage. The proposed strategy supposes that every node in the network has four sides. Simulation results illustrate that BCP achieves a high coverage ratio by replacing the border nodes with transfer nodes, but it has the extra overhead to find out the transfer nodes and then replace it. The recognition of the four sides of every node is the main drawback of BCP. The BDCIS and $\mathrm{BCP}$ are assisted as a comparison in the proposed work because these are an energy efficient routing hole detection algorithm with some deficiencies as discussed above.

Unfortunately, the time or space complexity of these existing algorithms is higher. The routing hole detection is necessary to avoid the long detour path, which is caused by forwarding packets via the hole boundary node. Hence, there is a dire need to detect a routing hole with less time and space complexity. A novel energy efficient routing hole detection (EEHD) is proposed for balanced energy consumption and less computational overhead.

\section{ENERGy EFFICIENT Routing Hole DETECTION (EEHD) ALGORITHMS}

In proposed work, the sensor nodes are considered to have limited energy and can sense their own residual energy and all the nodes of the sensor network are equipped with the same amount of energy level in the beginning. Base Station (BS) is without energy restriction, but far away from the area of sensor nodes. All sensor nodes are static and have the same capabilities, just a subset of deployed sensor nodes is designed as CHs. In this architecture, multi-hop clustering is applied to 
communicate with the BS. Sensor nodes sense the environment at a fixed rate and always have data to send to the BS. The energy required for transmitting a message is the same for all nodes because the radio channel is symmetric. It is required that every sensor node, with a unique identification number (ID), can monitor its own battery power level. $\mathrm{CH}$ performs data aggregation and sends the compressed data to BS via multi-hop as shown in Fig. 4.

The routing hole should be detected in advance before to start the data transportation phase, and the nodes placed on the boundary of the hole announced the hole information to its neighbor nodes. Hence the long detour path can be avoided.

Many detection techniques are presented in the literature which detects the boundary nodes to avoid the long detour path. But each has its weaknesses like computational overhead and maximum energy consumption. To avoid these drawbacks the proposed routing hole detection algorithm sets a threshold value for detecting the hole. $\mathrm{CH}$ mark itself as unavailable once its local energy fall behind a threshold. Then $\mathrm{CH}$ advertises itself as a critical node to its neighbor and re-clustering will take place. First, to start data transportation phase the routing hole is detected in advance, then the nodes located on the boundary of the hole advertises the hole information to its neighbor nodes. Hence the long detour route can be avoided for communication. The proposed routing hole detection algorithm detects the routing hole by the required energy threshold $E_{T h r}$. If the energy of $\mathrm{CH}$ is less than $E_{T h r}$, identified itself as routing hole and re-clustering will be done by the neighbor nodes of the hole. In order to heal the routing hole problem, the periodic re-clustering is necessary across all nodes.

According to EEHD flowchart shown in Fig. 6 before to start the transportation of data, each $\mathrm{CH}$ will calculate and compare its energy with the threshold value. The $\mathrm{CH}$ will advertise itself as a routing hole if its energy is equal or less than to the threshold.

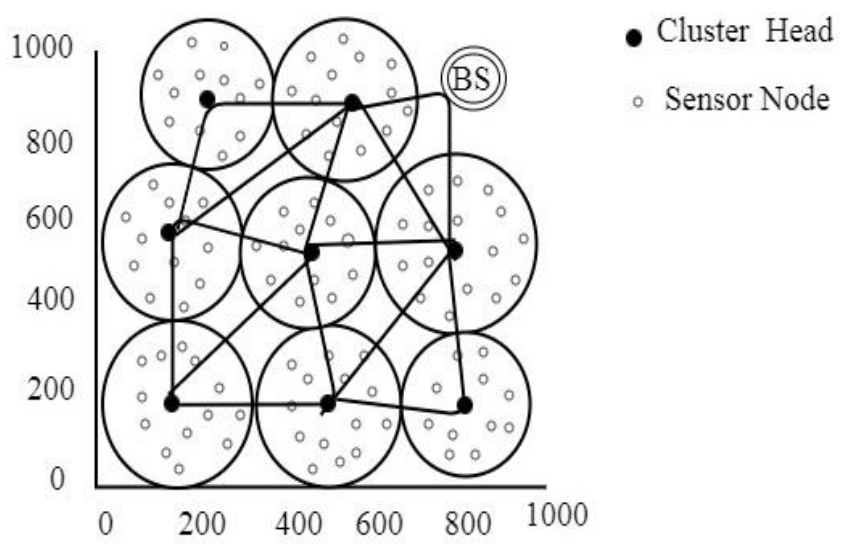

Fig. 4. Multi-Hop Clustering WSN.

\section{A. An Example Scenario of EEHD}

The routing hole detection algorithm can be better understood if we take the scenario for description. Let us take an example network of four CH's C1, C2, C3, and C4 as shown in Fig. 5.

The energy levels and member sensor nodes of each $\mathrm{CH}$ are given in Table I below. The energy level of CH's after every transportation is also given in Table I. The threshold value is supposed to be 1. After each transportation of packet, the $\mathrm{CH}$ will compare its energy with the threshold value if it's equal to 1 , the $\mathrm{CH}$ will advertise itself as a routing hole and trigger the re-clustering. As we observe in Table I that after 3rd iteration the cluster head C3 energy level is 1 which is equal to the threshold. Hence C3 will advertise itself as a routing hole.

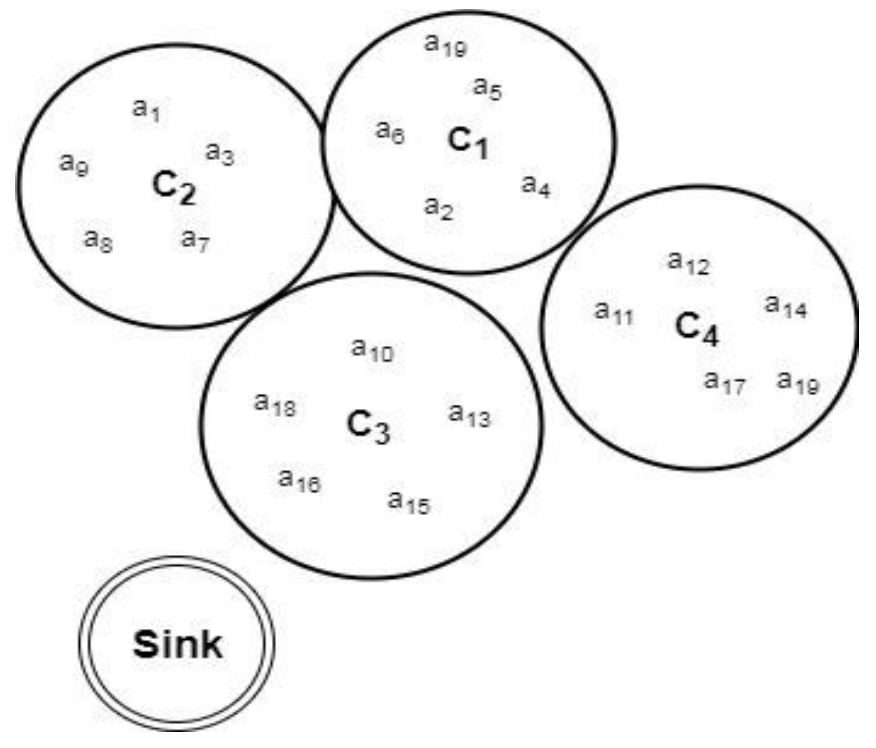

Fig. 5. EEHD Example Scenario.

TABLE I. ENERGY LEVEL OF CH'S

\begin{tabular}{|l|l|l|l|l|l|}
\hline & & Cluster Heads & Energy & Neighbors & \multicolumn{3}{|c|}{$\begin{array}{l}\text { CH energy at each } \\
\text { iteration of } \\
\text { transportation }\end{array}$} \\
\hline & & & \multicolumn{1}{|c|}{ st } & 2nd & 3rd \\
\hline C1 & 5 & a2, a4, a5, a6 & 4 & 3 & 2 \\
\hline C2 & 6 & a1, a3 a7, a8, a9 & 6 & 4 & 2 \\
\hline C3 & 4 & $\begin{array}{l}\text { a10, a13 a15, a16, } \\
\text { a18 }\end{array}$ & 4 & 3 & 1 \\
\hline C4 & 8 & a11, a12, a14,a17 & 8 & 7 & 5 \\
\hline
\end{tabular}




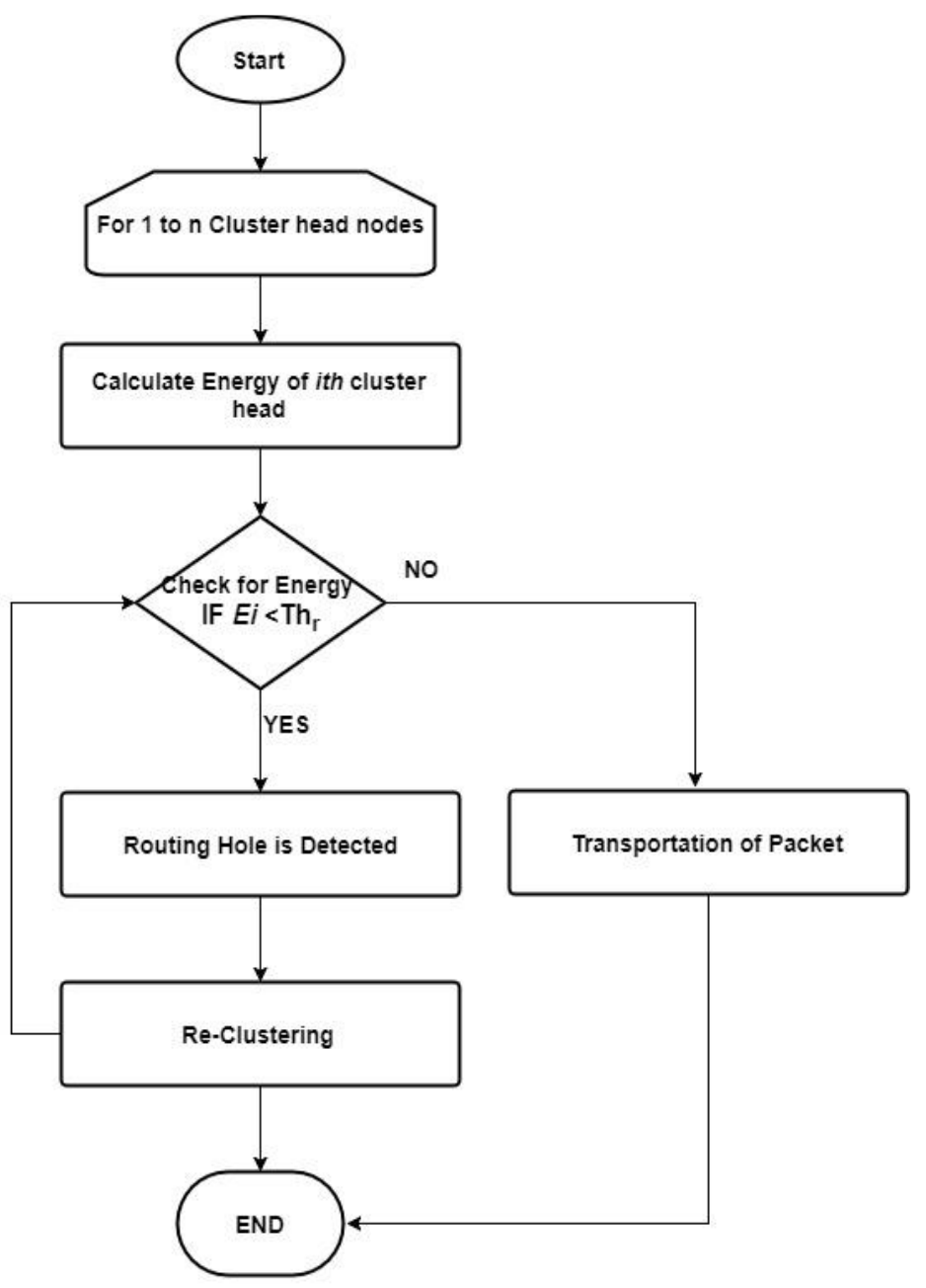

Fig. 6. Flowchart for Routing Hole Detection.

\section{PERFORMANCE DISCUSSION}

In this section, the performance of proposed EEHD is evaluated on the basis of computation overhead, hole detection time, energy consumption and detection rate of holes. The proposed algorithm is compared with other existing hole detection algorithms such as BCP [25] and BDCIS [24]. The proposed algorithm is evaluated by answering the following questions:

- Simulation Setup: To explore the performance of the proposed detection algorithm, we generate a sensor network comprising of randomly deployed nodes and carry out extensive simulations in MATLAB R2014b. Random data traffic was generated from the sensor nodes having a transmission range of 150 meters and the sink node was kept outside of the topology.

- Performance Metrics: For assessment of the performance of the proposed routing hole detection algorithm, a set of performance metrics is used such as detection time, energy consumption and computation overhead. For best performance, a strategy is needed that uses the sensor nodes energy in a uniform manner, with minimum detection time and less computation overhead.
The simulation results are compared with existing detection techniques on the above-mentioned performance metrics are shown in figures.

\section{A. Hole Detection Time}

The proposed routing hole detection algorithm is evaluated by hole detection time as compared to other existing hole detection strategies like BCP and BDCIS shown in Fig. 7. The hole detection time of the proposed routing hole detection algorithm is minimum as compared to BCP and BDCIS. The hole detection time of BCP is less than BDCIS because the computation overhead BDCIS is more than BCP. However, in all three protocols, the hole detection time is increased with the increase in the number of holes.

For 10 holes, the detection time of a BCP and BDCIS is about 4200 and 3900 simulation seconds respectively, and for the proposed EEHD algorithm, it is about 3000 simulation seconds. There is a considerable decrease as compared to other detection strategies. The same decrease of time can be observed for 20 holes, i.e. 1000 simulation seconds. And for 30 holes the detection time of the proposed EEHD algorithm decreases about 1300 simulation seconds. 


\section{B. Energy Consumption}

In this section, the energy consumption of proposed routing hole detection is compared with other detection techniques such as BCP and BDCIS as shown in Fig. 8. BCP has less energy consumption than BDCIS because every node receives only one message from its immediate neighbors to build its Localized Voronoi Polygons (LVP) and the minimum energy consumption is recorded for our proposed routing hole detection algorithm because it's don't need any computation like BDCIS or neighbor node information BCP.

For 10 holes, the energy consumption of a BCP and BDCIS is about 43 and 38 Joules while for the proposed EEHD algorithm, it is about 30 Joules. There is a considerable decrease in the energy consumption rate as compared to other detection strategies. The 10 Joules decrease in energy consumption can be noticed for 20 holes. As compared to BCP and BDCIS, for 30 holes the energy consumption rate of the proposed EEHD algorithm is decreased about 13 Joules.

\section{Computation Overhead}

The BCP has extra overhead to find out the transfer nodes and then replace with border nodes. Tow messages have to send by every node in BDCIS strategy, one for the neighbor node checking while the other contains the list of neighbor nodes. Hence, the computation overhead of BDCIS is more than BCP. It can be observed in Fig. 9 that our proposed EEHD algorithm has the lowest computation overhead because it just depends on one comparison of energy with the threshold.

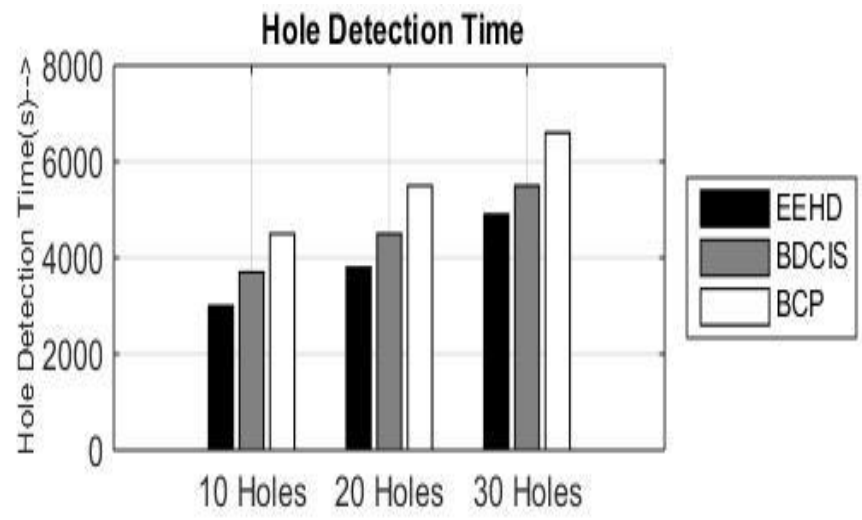

Fig. 7. Comparison of Routing Hole Detection Time.

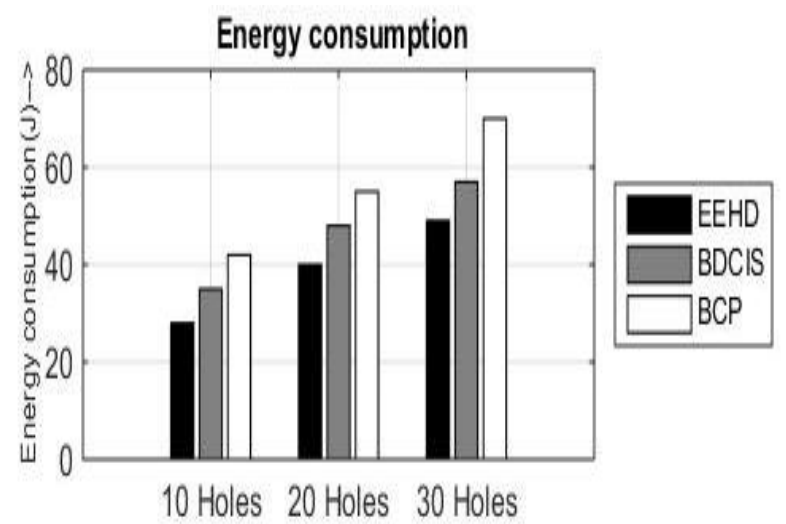

Fig. 8. Comparison of Average Energy Consumption.

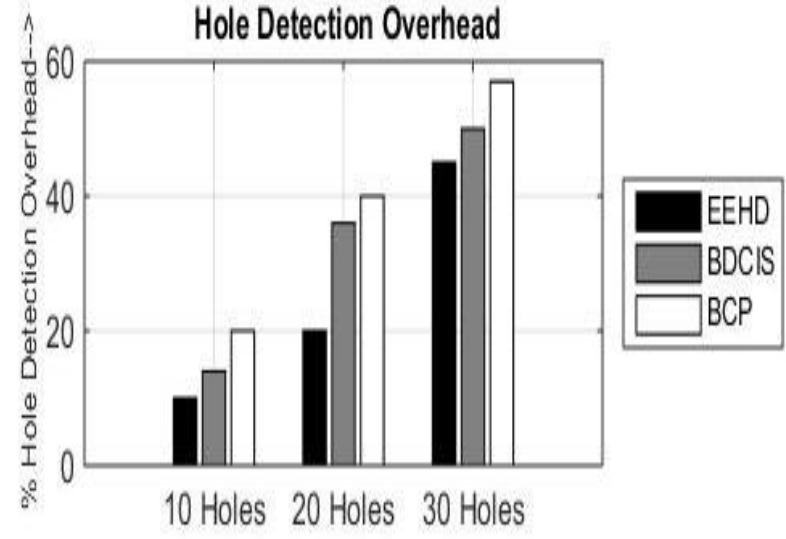

Fig. 9. Hole Detection Overhead.

For 10 holes, the detection overhead of proposed EEHD algorithm is $10 \%$ and $15 \%$ less from BDCIS and BCP, respectively. There is a considerable decrease in overhead as compared to other detection strategies. As compared to BCP and BDCIS, for 30 holes the overhead of the proposed EEHD algorithm is decreases by about $13 \%$.

\section{CONCLUSION}

An energy efficient routing hole detection algorithm is proposed in which the energy of each $\mathrm{CH}$ is compared with threshold if the energy level of $\mathrm{CH}$ is equal to the threshold, $\mathrm{CH}$ will advertise itself as routing hole and member nodes will call to the re-clustering algorithm. Prior to the detection of the holes, it is assumed that the construction of clusters has been performed.

Simulation results show enhancement of the proposed detection algorithm in the comparison of existing routing holes detection techniques, such as BDCIS and BCP. It is proven that the proposed EEHD algorithm able to detect holes with less time and computational complexity, thus consumed minimum energy.

\section{REFERENCES}

[1] Y. Ge, S. Wang, and J. Ma, "Optimization on TEEN routing protocol in a cognitive wireless sensor network," EURASIP Journal on Wireless Communications and Networking, vol. 2018, p. 27, February 012018.

[2] N. Ahmed, S. S. Kanhere, and S. Jha, "The holes problem in wireless sensor networks: a survey," SIGMOBILE Mob. Comput. Commun. Rev., vol. 9, pp. 4-18, 2005.

[3] G. Ahmed, N. M. Khan, and M. M. Y. Masood, "A dynamic transmission power control routing protocol to avoid network partitioning in wireless sensor networks," in Information and Communication Technologies (ICICT), 2011 International Conference on, 2011, pp. 1-4.

[4] P. Kumar Sahoo, M.-J. Chiang, and S.-L. Wu, "An Efficient Distributed Coverage Hole Detection Protocol for Wireless Sensor Networks," Sensors (Basel, Switzerland), vol. 16, p. 386, 03/17 12/21/received 03/11/accepted 2016.

[5] P. Antil and A. Malik, "Hole Detection for Quantifying Connectivity in Wireless Sensor Networks: A Survey," Journal of Computer Networks and Communications, vol. 2014, p. 11, 2014.

[6] H. Huang, H. Yin, G. Min, X. Zhang, W. Zhu, and Y. Wu, "Coordinate-Assisted Routing Approach to Bypass Routing Holes in Wireless Sensor Networks," IEEE Communications Magazine, vol. 55, pp. 180-185, / 2017.

[7] A. Debnath and M. K. Debbarma, "Energy efficient-dynamic and self-optimized routing in a wireless sensor network based on ant colony 
optimization," International Journal of Networking and Virtual Organisations, vol. 20, pp. 184-194, 2019.

[8] J. Wang, Y. Gao, W. Liu, A. K. Sangaiah, and H.-J. Kim, "An Improved Routing Schema with Special Clustering Using PSO Algorithm for Heterogeneous Wireless Sensor Network," Sensors, vol. 19, p. 671, 2019.

[9] K. A. Darabkh, M. Z. El-Yabroudi, and A. H. El-Mousa, "BPA-CRP: A balanced power-aware clustering and routing protocol for wireless sensor networks," Ad Hoc Networks, vol. 82, pp. 155-171, 2019.

[10] A. V. Bharathy and V. Chandrasekar, "A Novel Virtual Tunneling Protocol for Underwater Wireless Sensor Networks," in Soft Computing and Signal Processing, ed: Springer, 2019, pp. 281-289.

[11] J. G. Q. Fang, and L. Guibas., " "Locating and bypassing routing holes in sensor networks"," Mobile networks and applications, vol. 11, pp. 187-200, 2006.

[12] P. K. Sahoo and L. Wei-Cheng, "HORA: A Distributed Coverage Hole Repair Algorithm for Wireless Sensor Networks," Mobile Computing, IEEE Transactions on, vol. 14, pp. 1397-1410, 2015.

[13] J. Yu, Y. Qi, G. Wang, and X. Gu, "A cluster-based routing protocol for wireless sensor networks with nonuniform node distribution," AEU International Journal of Electronics and Communications, vol. 66, pp. 54-61, 2012.

[14] A. U. Rahman, A. Alharby, H. Hasbullah, and K. Almuzaini, "Corona-based deployment strategies in wireless sensor network: A survey," Journal of Network and Computer Applications, vol. 64, pp. 176-193, 2016.

[15] H. A. Hashim, B. O. Ayinde, and M. A. Abido, "Optimal placement of relay nodes in wireless sensor network using artificial bee colony algorithm," Journal of Network and Computer Applications, vol. 64, pp. 239-248, 2016.

[16] C. Sha, H. Chen, C. Yao, Y. Liu, and R.-c. Wang, "A Type of Energy Hole Avoiding Method Based on Synchronization of Nodes in Adjacent
Annuluses for Sensor Network," International Journal of Distributed Sensor Networks, vol. 2016, p. 14, 2016.

[17] P. Kumar Sahoo, M. J. Chiang, and S. L. Wu, "An Efficient Distributed Coverage Hole Detection Protocol for Wireless Sensor Networks," Sensors, vol. 16, 2016.

[18] W. An, N. Qu, F.-M. Shao, X. Xiong, and S. Ci, "Coverage hole problem under sensing topology in flat wireless sensor networks," Wireless Communications and Mobile Computing, vol. 16, pp. 578-589, 2016.

[19] K. Latif, N. Javaid, A. Ahmad, Z. A. Khan, N. A. Alrajeh, and M. I. Khan, "On energy hole and coverage hole avoidance in underwater wireless sensor networks," IEEE Sensors Journal, vol. PP, pp. 1-1, 2016.

[20] K. N. Kannan and B. Paramasivan, "Development of Energy-Efficient Routing Protocol in Wireless Sensor Networks Using Optimal Gradient Routing with On Demand Neighborhood Information," International Journal of Distributed Sensor Networks, vol. 2014, p. 7, 2014.

[21] M. M. Umar, N. Alrajeh, and A. Mehmood, "SALMA: An Efficient State-Based Hybrid Routing Protocol for Mobile Nodes in Wireless Sensor Networks," International Journal of Distributed Sensor Networks, vol. 2016, p. 11, 2016.

[22] J. Yang, Z. Fei, and J. Shen, "Hole detection and shape-free representation and double landmarks based geographic routing in wireless sensor networks," Digital Communications and Networks, vol. 1, pp. 75-83, 2015/02/01/2015.

[23] A. Zahedi and F. Parma, "An energy-aware trust-based routing algorithm using gravitational search approach in wireless sensor networks," Peer-to-Peer Networking and Applications, pp. 1-10, 2019.

[24] R. Beghdad and A. Lamraoui, "Boundary and holes recognition in wireless sensor networks," Journal of Innovation in Digital Ecosystems, vol. 3, pp. 1-14, 2016/06/01/ 2016.

[25] A. Dabba and R. Beghdad, "BCP: A Border Coverage Protocol for wireless sensor networks," in Science and Information Conference (SAI), 2014, 2014, pp. 632-640. 